\title{
Anaphylaxis-Induced Atrial Fibrillation and Anesthesia: Are We Missing Something?
}

\author{
Minati Choudhury ${ }^{1}$ Poonam Malhotra Kapoor ${ }^{1}$ \\ ${ }^{1}$ Department of Cardiac Anaesthesia, Cardiothoracic Sciences \\ Centre, All India Institute of Medical Sciences, New Delhi, India
}

J Card Crit Care TSS 2020;3:51-52

\section{Brief Communication}

Atrial fibrillation (AF) represents a complex cardiac arrhythmia, which is related to various risk factors, such as aging, allergy, oxidative stress, hypertension, cardiovascular diseases (CVD), diabetes mellitus or thyrotoxicosis. ${ }^{1}$ recently, several reports have been published associating atrial fibrillation with anaphylactic reaction from food consumption, mitochondrial dysfunction. All these causative factors are interrelated and can exist in the same patients. ${ }^{2}$ The relationship between anaphylaxis and cardiovascular event is important. It is also true that fatal cardiovascular events (atrial or ventricular fibrillation) represent the leading cause of death in anaphylaxis.

The heart is the primary source and target of several mediators released during anaphylaxis. The release of these mediators influences heart rate (HR), coronary tone, and ventricular function. Some of the commonly used drugs during the perioperative period, for example, $\beta$-lactam antibiotics, anaesthetics, $\mathrm{N}$-acetylcysteine, and antiarrhythmic drugs itself are also noted to be responsible for the development of AF during anaphylaxis. ${ }^{2}$ Diseases of cardiovascular system also increases the risk of catastrophic anaphylaxis as well as AF, for example, mast cell mediates acute coronary syndrome and drugs used for CVD treatment, especially ACE inhibitors and $\beta$-blockers possibly exacerbate anaphylaxis or make it more complicated to manage. ${ }^{2}$ What is more important to know in such condition is that, which should be first, the treatment of several other common symptoms of anaphylaxis or AF ? Moreover, when all the etiologies present in the same patient, at times, it is difficult to treat the AF.

Control of HR and prevention of complications of AF, for example, hypotension and heart failure, are essential. The American Heart Association/Heart Rhythm Society recommends intravenous $\beta$-blocker or calcium channel blockers especially the nondihydropyridine group of drugs (verapamil or diltiazem) to reduce the ventricular rate in the presence of sudden AF in the absence of an accessory pathway. ${ }^{3}$ Amiodarone as a class I recommendation in the presence of

\begin{abstract}
Address for correspondence Minati Choudhury, MD, PGDip, Department of Cardiac Anaesthesia, Cardiothoracic Sciences Centre, All India Institute of Medical Sciences, Room No.10, New Delhi 110 029, India (e-mail: minatichoudhury2002@yahoo.co.in).
\end{abstract}

heart failure with AF without accessory pathway and a class IIa recommendation for control of $\mathrm{HR}$ in $\mathrm{AF}$ if other routinely used control agents failed or contraindicated. Amiodarone is the most frequently used agent for AF despite it harboring an extensive side effect. In case of isolated AF, $\beta$ blocker may be tried first but sotalol, propafenone, and flecainide are particularly effective. Esmolol at a dose of $500 \mu \mathrm{g} / \mathrm{kg}$ over 1 minute followed by infusion at $50-200 \mu \mathrm{g} / \mathrm{kg} / \mathrm{min}$ is preferred due to its quick onset of effect and short duration of action. Diltiazem at a dose of $0.25 \mathrm{mg} / \mathrm{kg}$ followed by $5-15 \mathrm{mg} / \mathrm{h}$ infusion is also effective in the emergency scenario. However, both $\beta$ blocker and CCB are associated with the risk of hypotension, heart block, and heart failure. Beta blockers are contraindicated in patients with asthma. Electrical cardioversion is recommended in hemodynamically unstable patients. Patient should be investigated for underlying coexisting problem if AF is persistent (more than 7 days). Treatment with $\mathrm{H} 1$ antagonist is controversial because these drugs slow atrioventricular conduction and inhibit ventricular fibrillation, and they are considered to be safe in $\mathrm{AF}^{4}$ Corticosteroids help during oxidative stress, and no contraindication have been described in the existing literature in case of AF. The benefits of antioxidant therapy using vitamins $C$ and $E$, polyunsaturated fatty acid, statin, or nitric oxide donor in the prevention of AF are well described in various preclinical studies and clinical trials; however, the benefits of these agents during acute care settings are yet to be proved. ${ }^{3}$ In spite of the fact that, the genetic basis of origin of both anaphylaxis and AF has been essentially proved, and the application of this understanding in the management of patients with AF during anaphylaxis has not happened so far. Some evidence indicates that AF patients who are carriers of the common chromosome 4q25 single-nucleotide polymorphism (SNP) respond to pharmacological therapy differently from individuals without these SNPs. There is always a hesitation to utilize this genetic data to bedside. This is probably due to the fact that prospective clinical trial to validate the common AF risk alleles is not available. ${ }^{5}$
DOI https://doi.org/

10.1055/s-0039-1696906 ISSN 2457-0206.
Copyright (22020 Official

Publication of The Simulation

Society (TSS), accredited

by International Society of

Cardiovascular Ultrasound (ISCU).
License terms

$\Theta \odot \Theta \circledast$ 
Patients with acute coronary syndrome with developed anaphylaxis and AF need to be considered along with the benefits and potential harm of epinephrine which is considered to be the life-saving drug in acute care setting. One has to weigh the potential adverse effect of this drug against the possible death from untreated anaphylaxis. No absolute contraindication of the use of epinephrine during anaphylaxis is described elsewhere in literature.

Perioperative anaphylaxis is increasing as anesthesia protocols become more complex. Resultant AF may have lifethreatening consequences. Therefore, any suspected reaction must be extensively investigated to the root cause to provide precise recommendation for future anesthetic management.

\section{Financial Support}

None.

\section{Conflict of Interest}

None.

\section{References}

1 Bernstein DI, Wanner M, Borish L, Liss GM; Immunotherapy Committee, American Academy of Allergy, Asthma and Immunology. Twelve-year survey of fatal reactions to allergen injections and skin testing: 1990-2001. J Allergy Clin Immunol 2004;113(6):1129-1136
2 Triggiani M, Patella V, Staiano RI, Granata F, Marone G. Allergy and the cardiovascular system. Clin Exp Immunol 2008;153(Suppl 1):7-11

3 Fuster V, Rydén LE, Cannom DS, et al; American College of Cardiology/American Heart Association Task Force on Practice Guidelines; European Society of Cardiology Committee for Practice Guidelines; European Heart Rhythm Association; Heart Rhythm Society. ACC/AHA/ESC 2006 guidelines for the management of patients with atrial fibrillation: a report of the American College of Cardiology/American Heart Association Task Force on practice guidelines and the European Society of Cardiology Committee for practice guidelines (writing committee to revise the 2001 guidelines for the management of patients with atrial fibrillation): developed in collaboration with the European Heart Rhythm Association and the Heart Rhythm Society. Circulation 2006;114(7):e257-e354

4 Wasserman SI. The heart in anaphylaxis. J Allergy Clin Immunol 1986;77(5):663-666

5 Cho HC, Marbán E. Biological therapies for cardiac arrhythmias: can genes and cells replace drugs and devices? Circ Res 2010;106(4):674-685 